\title{
SOME UNUSUAL COMPLICATIONS OF HERPES ZOSTER
}

\author{
BY
}

\author{
C. W. M. WHITTY and A. M. COOKE
}

\author{
From the Radcliffe Infirmary, Oxford
}

Herpes zoster is a virus infection which attacks primarily the posterior nerve root ganglion and adjacent spinal grey matter. Head and Campbell (1900), though unaware of the causative agent, termed it an acute posterior poliomyelitis. However, more extensive involvement of brain and spinal cord, including motor neurones, may occur. It is an infection which usually gives prolonged immunity. Van Rooyen and Rhodes (1940) considered that recurrent zoster was extremely rare. When it does occur, attacks are usually separated by years. The three cases described below illustrate unusual clinical manifestations of the condition. All three had clinical evidence of myelitis situated at the central end of the nerves involved in the zoster. In two the infection pursued a migratory course, passing through several segmental skin zones with a few weeks' interval between the involvement of each. The third case illustrates the precipitation of a zoster infection by trauma to a nerve. Such " symptomatic" zosters are recognized, but in these cases the sequel of an encephalitis or myelitis, which occurred here, is not usual.

\section{Case Histories}

Case 1.-A widow, aged 73 years, in July, 1947, developed an unpleasant tingling feeling in the skin of the left buttock. Within forty-eight hours a typical herpetic rash appeared, which spread to involve the right buttock, so that she had a saddle-area herpes zoster of the third and fourth sacral segments. Four days later she developed urinary retention with overflow, for which she was admitted to hospital. This required catheterization for five days. Thereafter voluntary evacuation was possible, at first with the aid of manual pressure. When she left hospital sixteen days after the onset of the retention she was passing urine normally except for a slight stress incontinence that had been present previously. She remained well for about two weeks. Then, some five weeks after the first zoster eruption had appeared, there was a second outbreak, after the usual prodromal skin sensation, this time below the shoulder blades in the sixth dorsal and parts of the seventh dorsal areas. This outbreak was also bilateral. The rash cleared, leaving some residual burning pain in the area. One month later the left trigeminal nerve was affected, mainly in the second division, and in a few dayso this spread to involve the second and then the firsto division of the nerve on the right side. Thereafter she $\overline{\bar{c}}$. remained well until August, 1948, when she developed $\bar{\sigma}_{\bar{\sigma}}$ an isolated left facial palsy of peripheral type. No응 vesicles were found, and there was nothing to suggest that this was linked with her previous zoster. The cerebrospinal fluid was not examined. No contact with. chicken-pox was recalled.

Case 2.-A married woman, aged 58 years, had had an attack of herpes of the left buttock in 1937. In June, 1948, she developed a zoster infection of the left tri-0 geminal nerve. Vesicles were mostly in the second and $\vec{N}$ third division areas. She also complained of diplopia, in especially with lateral eye movements. About ten dafs later vesicles appeared on the right side of the neck an the second to fourth cervical segments. The condition cleared and she was well for about two weeks. At the time a few scattered vesicles formed on the left side of 3 the neck in the third and fourth cervical segmends. These subsided rapidly. Three to four weeks later developed severe itching in the area of all three divisio of the right trigeminal nerve. A few vesicles formed ance the first division area. During the following week she noted some drooping of the left and then of the righto eyelid, and diplopia in all directions for distant objects. Examination at this time showed bilateral ptosis, lefto more than right, some lateral nystagmus, with a tendencyo to skew deviation, and uncrossed diplopia present in all directions. There was marked hyperæsthesia throughout $\vec{F}$ the territory of the right trigeminal nerve. The pupils 3 were $3 \mathrm{~mm}$. in diameter, equal, central, and circular, and reacted to light and accommodation. No asymmetry of sweating or temperature could be felt in the face. There was no evidence of long tract involvement. The cerebrospinal fluid contained three lymphocytes per c.mm. and $40 \mathrm{mg}$. of protein per $100 \mathrm{c.cm}$.

A few days later she showed increased ptosis, complete on the left side, and developed an unsteady gait ando difficulty in starting micturition. Examination revealed an extensor plantar response on the right side, and an absent plantar response on the left. These features? persisted for a week, after which the ptosis cleared up, followed by the bladder symptoms and ataxia. In a․ fortnight's time the only remaining abnormalities weren diplopia on looking to the left and the plantar responses? as above. Three months after the onset of the second phase of her illness the patient was up and about normally, N but still complained of slight diplopia. 
Case 3.-A farm labourer, aged 43 years, was seen in January, 1948, suffering from severe trigeminal neuralgia of the second division on the right side, of six weeks' duration. There was a history of a previous attack eight months before, when extraction of carious teeth had been followed by cessation of pain. At the time of this first attack he had complained of unsteadiness of the right leg, though examination then showed no abnormality of the central nervous system. During the second attack also there was an indefinite history of ataxia on one occasion when the patient was walking in the dark, but again the nervous system was normal. In view of the limited zone of pain, an alcohol injection of the infraorbital nerve was done. This produced anzsthesia of the appropriate area and some reduction in the intensity of the pain. Three days later the patient developed a zoster eruption of the whole skin area of the second division of the right fifth nerve. Within a week of this incident signs of mid-brain involvement appeared and progressed rapidly. Examined at this time he had nystagmus, vertical as well as lateral, skew deviation with diplopia, slight dysarthria, and incoordination with intention tremor of right arm and leg movements. The cerebrospinal fluid showed $40 \mathrm{mg}$. of protein per $100 \mathrm{c.cm}$. and no cells. The signs subsided slowly over a period of six weeks. There was no history of exposure to chicken-pox.

\section{Discussion}

Three points are of interest in these cases. First, all three showed signs of myelitis accompanying the cutaneous zoster. This complication, known since the end of the last century, has been well recognized since the full discussion of it under the title "myelite zosterienne" by Lhermitte and Nicolas (1924). Some degree of localized posterior myelitis is probably frequent in these cases. It is shown clinically only if it extends to involve long tracts, or passes forwards to anterior horn cells. The histological picture, discussed by Head and Campbell (1900) has recently been elegantly demonstrated by Denny-Brown and others (1944).

Secondly, Cases 1 and 2 raise a problem in the development of immunity to the zoster virus. There is much clinical evidence-chicken-pox follows contact with herpes zoster, though the reverse is less common-which supports the immunological studies of Netter and Urbain (1926) on the identity of this organism with that causing chicken-pox. Immunity produced by an attack of either of these conditions is usually prolonged. Second attacks are rare, and if they occur they do so many years after the first (Hruszeck, 1934 ; Graham-Little, 1937). During an attack of zoster, vesicles may spread to other dermatomes than those first involved, or a generalized eruption may follow. In either case, this usually occurs during the first five days after the first eruption (Weber, 1916 ; Brain, 1931), though cases are mentioned where new blebs appear up to ten or eleven days later, and in one a fresh eruption in the originally affected zone seven weeks later (Schiff and Brain, 1930 ; Denny-Brown and others, 1944), while Bokay (quoted by Brain, 1931) recorded a generalized eruption following as long as three weeks after a local zoster. Presumably after this interval, which in most cases is of a few days' duration, the ultimately long-lasting immunity has been established. The mode of production of such immunity in certain virus diseases is in doubt. One view is that after the initial infection the virus maintains itself within the cells of the host. Its rate of reproduction is adjusted so that enough is formed to infect the host cell and any daughter cells derived from it, but not enough to cause clinical manifestations of the disease. A state of true symbiosis is thus achieved. In some diseases, notably herpes simplex, the balance between virus growth and cell function can be easily upset by some general infection or even local trauma, with the production of clinical herpes simplex. In other conditions, of which herpes zoster and chicken-pox are examples, the virus/host cell symbiosis is assumed to be more stable. Second attacks of these diseases are supposed to occur by reinfection after the virus from the first attack has died out.

However, in the two cases discussed here such a sequence of events did not seem to occur. No effective immunity was established by the attacks, at any rate for two or three months. This clinical course is more reminiscent of a herpes simplex than of a zoster infection, and this possibility must be considered. The presence of central nervous system involvement does not exclude such an interpretation, as this complication with herpes simplex has been recorded sporadically during the last 45 years (Howard, 1905; Whitman and others, 1946), severe and even fatal encephalitis being noted (Swan, 1943). If this be the explanation, then it must be recognized that a vesicular eruption of dermatome distribution and with other clinical characters of a zoster may in fact be a herpes simplex. Alternatively, something may have prevented or delayed the development of normal immunity to the zoster virus, in which case it must be borne in mind that immunological behaviour of the type shown in these cases is not incompatible with infection by this virus. The solution to the problem would be given by identification of the causal agent. Antigen/antibody studies in such cases are complicated by the fact that 50 per cent. of the population, usually with a history of recurrent herpes simplex, have circulating antibodies to this virus (Andrewes and Carmichael, 1930), so that their presence in a given case is no proof that a herpetic eruption is necessarily due to the simplex 
virus. Direct isolation of the virus from the cerebrospinal fluid or vesicles would probably be necessary to provide an unequivocal answer.

Thirdly, Case 3 exemplifies a "symptomatic" zoster, secondary to peripheral trauma of the fifth cranial nerve. This is not uncommon after involvement of the first sensory neurone, especially at root level, by secondary neoplasm or tuberculous granuloma. Its cause is generally supposed to be the zoster virus, trauma being only a precipitating factor (Brain, 1931). Such a view is supported in this case by the sequel of mid-brain encephalitis, inexplicable on a purely traumatic ætiology. A further question arises here. Could the mid-brain signs represent an exacerbation of a pre-existing mid-brain lesion of which the original tic douloureux was itself a symptom? Such a lesion might be a latent virus encephalitis whose symbiotic balance was upset by trauma to the fifth nerve, or it might be a separate lesion aggravated by infection of the fifth nerve ganglion and its central connexions. In our case the known absence of pre-existing mid-brain signs supports the simpler explanation that the encephalitis depended on the preceding zoster infection. The question arises to some extent in any case of this condition with central nervous system complications, especially if they are distant from the segmental area first involved. This has been discussed at some length by Schiff and Brain (1930). Pre-existing infection has to be considered also when zoster follows root section in the treatment of an apparently " idiopathic" trigeminal neuralgia, a not very uncommon sequence. The answer must await further knowledge of the behaviour of neurotropic viruses.

\section{Summary}

Three cases of herpes zoster are described. All $_{\mathbb{Q}}^{Z}$

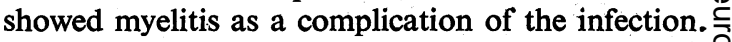
Two had recurrent attacks of zoster in different of skin zones over a period of two or three months, thus showing a marked difference from the usual $D$ behaviour of the zoster virus, which confers life-long immunity by a single attack. The possibility that these were really cases of herpes simplex is mentioned. The third case had a zoster infection pre-. cipitated by injury to the fifth nerve. The relation $\overrightarrow{\vec{B}}$ of virus infection to such precipitating factors is briefly discussed.

\section{REFERENCES}

Andrewes, C. H., and Carmichael, E. A. (1930). Lancet, $1,857$.

Brain, W. R. (1931). Brit. med. J., 1, 81.

Denny-Brown, D. E., Adams, R. D., and Fitzgerald, P. J. (1944). Arch. Neurol. Psychiat., Chicago, 51, 216. Graham-Little, E. (1937). Brit. med. J., 1, 498.

Head, H., and Campbell, A. W. (1900). Brain, 23, 353. $\vec{N}$ Howard, W. T. (1905). Amer. J. med. Sci., 130, 1012. T Hruszeck, H. (1934). Derm. Wschr., 98, 515.

Lhermitte, J., and Nicolas, M. (1924). Rev. Neuro⿸广⿱乛龰 311, 361 .

Netter, A., and Urbain, A. (1926). C. R. Soc. BioR 윽 Paris, 94, 98.

Schiff, C. I., and Brain, W. R. (1930). Lancet, 2, 70.

Swan, C. (1943). Med. J. Aust., 1, 437.

Van Rooyen, C. E., and Rhodes, A. J. (1940). “Vir Diseases of Man." Oxford University Press. (Lofì don).

Weber, F. P. (1916). Internat. Clin. (Series 26), 3, 185.

Whitman, L., Wall, M. J., and Warren, J. (1946). Amer. med. Ass., 131, 1408. 